\title{
Atlas of tissue-specific and tissue-preferential gene expression in ecologically and economically significant conifer Pinus sylvestris
}

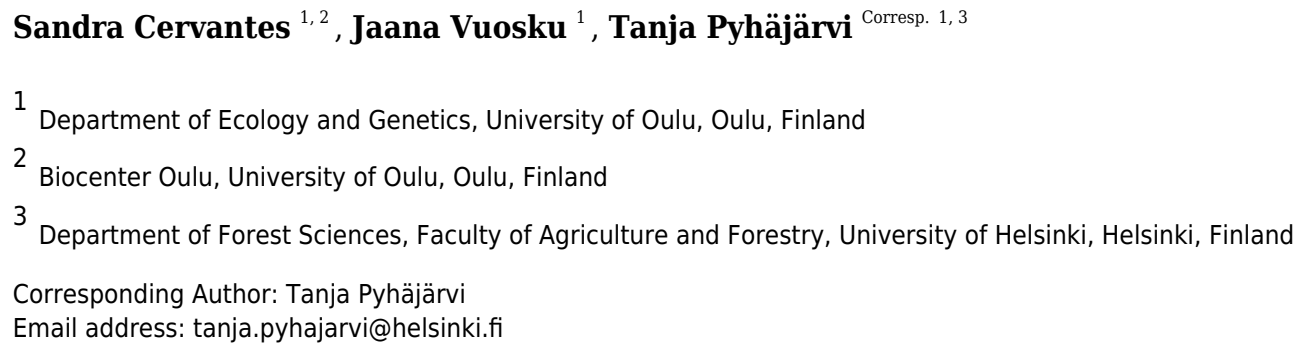

Despite their ecological and economical importance, conifers genomic resources are limited, mainly due to the large size and complexity of their genomes. Additionally, the available genomic resources lack complete structural and functional annotation.

Transcriptomic resources have been commonly used to compensate for these deficiencies, though for most conifer species they are limited to a small number of tissues, or capture only a fraction of the genes present in the genome. Here we provide an atlas of gene expression patterns for conifer Pinus sylvestris across five tissues: embryo, megagametophyte, needle, phloem, and vegetative bud. We used a wide range of tissues and focused our analyses on the expression profiles of genes at tissue level. We provide comprehensive information of the per-tissue normalized expression level, indication of tissue preferential upregulation and tissue-specificity of expression. We identified a total of 48,001 tissue preferentially upregulated and tissue specifically expressed genes, of which $28 \%$ have annotation in the Swiss-Prot database. Even though most of the putative genes identified do not have functional information in current biological databases, the tissuespecific patterns discovered provide valuable information about their potential functions for further studies, as for example in the areas of plant physiology, population genetics, and genomics in general. As we provide information on tissue specificity at both diploid and haploid life stages, our data will also contribute to the understanding of evolutionary rates of different tissue types and ploidy levels. 
1 Research article

2

3 Title: Atlas of tissue-specific and tissue-preferential gene expression in ecologically and

4 economically significant conifer Pinus sylvestris

5

6 Authors

7 Sandra Cervantes ${ }^{1,2}$, Jaana Vuosku$^{1}$, Tanja Pyhäjärvi*1,3

8 1Department of Ecology and Genetics, University of Oulu, Finland; ${ }^{2 B i o c e n t e r ~ O u l u, ~}$

9 University of Oulu, Finland; ${ }^{3}$ Department of Forest Sciences, Faculty of Agriculture and

10 Forestry, University of Helsinki, Finland.

11

12

13

$14{ }^{*}$ Corresponding author

15 tanja.pyhajarvi@helsinki.fi

16

17

18

19

20

21

22 


\section{Acknowledgments}

24 The authors wish to acknowledge CSC - IT Center for Science, Finland, for

25 computational resources and Dorota Paczesniak for the drawings of the main figures

26 included in this paper.

\section{Abstract}

28 Despite their ecological and economical importance, conifers genomic resources are limited, mainly due to the large size and complexity of their genomes. Additionally, the available genomic resources lack complete structural and functional annotation. Transcriptomic resources have been commonly used to compensate for these deficiencies, though for most conifer species they are limited to a small number of tissues, or capture only a fraction of the genes present in the genome. across five tissues: embryo, megagametophyte, needle, phloem, and vegetative bud. We used a wide range of tissues and focused our analyses on the expression profiles of genes at tissue level. We provide comprehensive information of the per-tissue normalized expression level, indication of tissue preferential upregulation and tissuespecificity of expression. We identified a total of 48,001 tissue preferentially upregulated and tissue specifically expressed genes, of which $28 \%$ have annotation in the SwissProt database. Even though most of the putative genes identified do not have functional information in current biological databases, the tissue-specific patterns discovered provide valuable information about their potential functions for further studies, as for

44 example in the areas of plant physiology, population genetics, and genomics in general.

45 As we provide information on tissue specificity at both diploid and haploid life stages, 
46 our data will also contribute to the understanding of evolutionary rates of different tissue

47 types and ploidy levels.

48 Keywords: Pinus sylvestris, RNA-seq, tissue-specific gene expression, conifer, 49 transcriptomics, megagametophyte.

\section{Introduction}

Conifers, a clade within the gymnosperms, represent a group of plants with significant economic and ecological relevance (Farjon, 2008). Several coniferous trees,

53 for example in Pinus and Picea genera are among the most important sources of wood

54 and timber, (San-Miguel-Ayanz et al., 2016; FAO Yearbook of Forest Products, 2020).

55 Conifers dominate boreal forests worldwide and can form large forested areas hosting a 56 variety of ecosystems. Furthermore, conifer forests are one of the major ecosystem

57 services providers and they are crucial for carbon sequestration (Bonan, Chapin \&

58 Thompson, 1995; DeAngelis, 2008; San-Miguel-Ayanz et al., 2016; Boonstra et al., 2016). Despite their importance, genomic resources for conifers, and gymnosperms in general, lag behind in availability compared to angiosperms. Although several contributions have been made recently to fill this gap (Nystedt et al., 2013; Birol et al., 2013; Zimin et al., 2014; Stevens et al., 2016; Mosca et al., 2019), conifer genome annotation remains a challenge, with both structural and functional annotations being far from perfect (Wegrzyn et al., 2014; Cañas et al., 2019). Conifer genomics resources are limited due to the large size of their genomes, ranging from 8 to $70 \mathrm{Gbp}$ (Zonneveld, 2012) and to the large number of repetitive elements (approximately $80 \%$ ) within them

67 (Nystedt et al., 2013; Neale et al., 2014; De La Torre et al., 2020). Proper and complete 68 annotation of the conifer genomes has also been complicated by the presence of long 69 introns (Nystedt et al., 2013; Wegrzyn et al., 2014), which prevents the routine use of 
70 common annotation software. Moreover, analyses of ortholog genes across different

71 species indicate that there are several gene groups which are unique to conifers or

72 conifer species specific, with no well-defined homologs in any of the angiosperm plant

73 models (Nystedt et al., 2013; Wegrzyn et al., 2014; Neale et al., 2014; Baker et al., 74 2018).

75 Transcriptomic resources have been particularly important for research in 76 conifers and other non-model species, as a strategy to compensate for the challenges

77 associated with efficient genome assembly and annotation (Cañas et al., 2019;

78 Wegrzyn et al., 2020). As the biological functions can not be directly inferred from nucleotide sequences, reference transcriptomes and gene expression studies are useful 80 in the identification and annotation of genes (Raherison et al., 2012; Wegrzyn et al., 81 2014; Merino et al., 2016; Little et al., 2016; Cañas et al., 2017). Transcriptome 82 information can also be used in conifers that lack reference genomes, as this 83 information can be used in the design of reduced genome representation targets

84 (Rellstab et al., 2019; Tyrmi et al., 2020). In addition to this, RNA-seq analyses allow the 85 identification of expression patterns and expression levels, which are essential components of evolutionary genomics studies. For example, selective constraints in

87 genes can be inferred from their expression patterns, as both breadth and expression 88 level are known determinants of evolutionary rates (Wright et al., 2004; Slotte et al., 2011). Selective constraints are also expected to differ between haploid and diploid 90 tissues which differ in the relative rate of expression, as tissue specificity and ploidy has

91 potentially drastic effects on the dynamics of e.g. purifying selection (Otto, Scott \& 92 Immler, 2015). 
Here we give a first glimpse of the expression patterns of tissue preferentially

94

95

upregulated (PUR) and tissue specifically expressed genes across five organs (embryo, megagametophyte, needle, phloem, and vegetative bud, hereafter called tissues, but see discussion) of Pinus sylvestris. $P$. sylvestris is a widely distributed conifer of large economic and ecological importance in Northern Eurasia (Pyhäjärvi, Kujala \& Savolainen, 2020). P. sylvestris is one of the main sources of timber and raw material for the pulp and paper industry in Europe and is a dominant species in boreal forests, with an estimated coverage area of 145 millions hectares (Pyhäjärvi, Kujala \& Savolainen, 2020). P. sylvestris is also a suitable model to answer evolutionary and genetic questions, especially regarding gymnosperm reproductive biology, its evolution and genetic consequences. For example, in conifers the maternal nuclear haplotype of an embryo is identical to the megagametophyte's nuclear haplotype (Williams, 2008), which makes it possible to separate expression of paternal and maternal haplotypes and alleles in the embryo (Verta, Landry \& MacKay, 2016).

Despite its importance and potential, $P$. sylvestris still lacks a reference genome, and currently there are limited genomic resources for this species (see however Wachowiak et al., 2015; Merino et al., 2016; Li et al., 2017; Höllbacher et al., 2017; Ojeda et al., 2019; Perry et al., 2020). To date, the few transcriptomic studies of $P$. sylvestris have been based on a small number of tissue types such as needles or seed tissues (Wachowiak et al., 2015; Merino et al., 2016). Identification of tissue preferentially upregulated and tissue specific genes is relevant because 1) understanding the patterns of expression across different kinds of tissues can aid to elucidate the organization of transcriptomes (Raherison et al., 2012). 2) Knowing the 
116 different profiles of expression across tissues can set the ground for evolutionary

117 analysis, as it is known from studies in mammals and angiosperms that the evolution of

118 gene expression differs across tissues and organs (Brawand et al., 2011; Yang \&

119 Wang, 2013). Ultimately this knowledge will help to gain a deeper understanding of the

120 determinants and main factors that affect the rate of adaptive evolution and the

121 dynamics at the genome level.

122 In this study we 1) provide a comparative transcriptomic resource for $P$. sylvestris

123 describing the expression level in five different tissues, 2) identify genes that are tissue

124 preferentially upregulated and tissue specifically expressed in each of the five tissues,

125 3) provide quantitative measures of tissue-specific expression for each gene per tissue

126 combination, and 4) conduct gene ontology enrichment analysis for each tissue type.

127 Our results are important for future studies in comparative conifer genomics, plant

128 physiology, population genetic analyses, evolutionary genetic studies, further gene

129

130

131

132

133 Materials and Methods

\section{Plant material and RNA sequencing}

135 We used the RNAseq data (trimmed reads, BioProject PRJNA531617) previously used

136 to assemble multiple reference transcriptomes of $P$. sylvestris by Ojeda et al. (2019).

137 The plant material was obtained during the growing season of 2016 (May 26th-27th)

138 and consisted of needles, phloem, vegetative buds, and seeds from six non-related 
139 adult Pinus sylvestris trees. We used data from trees growing in a natural forest

140 population to ensure that the observed patterns of tissue preferential expression and

141 specificity were robust to genotypic and environmental variation. The material was

142 collected at the Punkaharju Intensively Studied Site (ISS)

143 (http://www.evoltree.eu/index.php/intensive-study-sites) in Southern Finland (Table S1).

144 Samples were collected in collaboration with Natural Forest Research Institute Finland

145 LUKE that has an agreement on the forest research use with the owner Metsähallitus

146 (The Finnish Forest Administration). In Finland P. sylvestris is not endangered and

147 Finland does not regulate its genetic resources under Nagoya Protocol so CITES was

148 not applied and prior informed consent was not needed. The samples were collected

149 and identified by Tanja Pyhäjärvi. There is no voucher available for the specimens.

150 Relevant experimental procedures of Ojeda et al. (2019) are briefly summarized

151 here. Megagametophyte and embryo tissues were obtained by dissecting mature seeds

152 collected from the same mother trees from which the vegetative tissues were obtained.

153 Seeds were stored in the dark at $4^{\circ} \mathrm{C}$ until germination was induced by exposure to

154 moisture and continuous light for $48 \mathrm{~h}$. Total RNA was extracted from needle, bud, and

155 phloem using the Spectrum Plant Total RNA Kit (Protocol B, Sigma), followed by mRNA

156 capture with the NEBNext $($ Poly $(A)$ mRNA Magnetic Isolation Module (New England

157 Biolabs Inc.). For embryo and megagametophyte, mRNA was directly extracted from

158 the whole tissues with Dynabeads mRNA Direct Micro Kit (Thermo Fisher Scientific).

159 RNA concentration was quantified with Qubit RNA HS Assay kit (Thermo Fisher

160 Scientific). The quality and integrity of the RNA was visually assessed with a 2100

161 Bioanalyzer using the RNA 6000 Pico kit (Agilent). A total of 30 libraries were prepared 
162 by using the NEBNext Ultra Directional RNA Library Prep Kit for Illumina (New England

163 Biolabs Inc.). Sequencing (2 150 bp) was conducted with an Illumina NextSeq 500 at

164 the Biocenter Oulu Sequencing Centre (Oulu, Finland).

165 Transcript quantification and abundance matrices construction

166 We followed the Trinity Post-Transcriptome Assembly Downstream Analyses pipeline

167 (Trinity v.2.6.6) (Haas et al., 2013,

$168 \mathrm{https}$ //github.com/trinityrnaseq/trinityrnaseq/wiki/Trinity-Transcript-Quantification) to

169 generate quantification files at isoform level, and raw counts and normalized count

170 matrices at putative-gene level (hereafter referred as gene level matrices). For transcript

171 quantification we used the trimmed reads from Ojeda et al. (2019) and as reference we

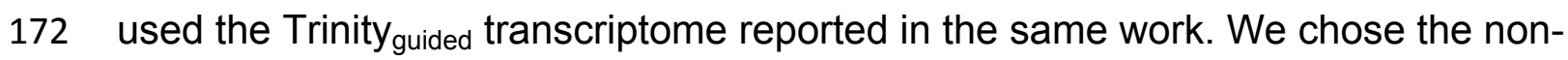

173 reduced redundancy Trinity guided $_{\text {transcriptome as reference (instead of the redundancy }}$

174 reduced assembly) to avoid mapping of reads from different paralogous genes to the

175 same contig. P. sylvestris has a large amount of paralogous and repetitive regions, and

176 with this we reduced the amount of false mapping across paralogs due to sequence

177 similarity.

178 To obtain independent transcript abundance estimates of each of the six

179 individuals in each of the five tissues we used Salmon 0.9.1 (Patro et al., 2017) as

180 implemented in the Trinity pipeline, with the --SS_lib_type (strand specific) and --

181 trinity_mode options. The --trinity_mode option generated a transcript-to-gene map that

182 allowed the estimation of counts from isoforms to generate counts at a putative gene

183 level during the count matrix generation step. Before any further analysis, we checked

184 for the presence of possible contaminants by searching contigs that had hits to the 
185 keywords 'alveolata', 'metazoa', 'fungi', 'bacteria', and 'archaea'. We search for exact 186 matches to these keywords from the results of a translated blast (BLASTX) of the 187 transcriptome annotation file (Ojeda et al., 2019; Ojeda, 2020). We then combined our 188 list of putative contaminants with the contaminants and organelles contigs lists reported 189 in Ojeda et al. (2019), and excluded them from the isoform quantification files and the 190 gene_trans_map. Contaminants were removed after the transcript quantification stage 191 to avoid the false mapping of contaminant reads to non-contaminant contigs in the 192 reference transcriptome.

193 We built three count matrices at the gene level based on the clean independent 194 transcript quantification estimates with the script abundance_estimates_to_matrix.pl 195 from the Trinity pipeline. For this, we generated a gene level raw counts matrix (Table 196 S2 available at

197 https://figshare.com/articles/dataset/Table_S2_raw_counts_matrix_txt/13121858), 198 which was then used to construct a transcript per million length normalized gene count 199 matrix (TPM escalated matrix) (Table S3 available at 200 https://figshare.com/articles/dataset/Table_S16_TPM_not_cross_norm_matrix_txt/1312 201 1870). The TPM escalated matrix accounts for differences in isoform lengths that 202 otherwise could inflate FDR due to differential transcript usage (Soneson, Love \& 203 Robinson, 2015). Finally, the TPM escalated matrix was used to construct a gene 204 counts matrix normalized using the Trimmed Mean of M values (TMM) method (Table 205 S4 available at

206 https://figshare.com/articles/dataset/Table_S17_TMM_EXPR_matrix_txt/13121873),

207 which accounts for differences in the distribution of transcript expression that could lead 
208 to an increase in false positive rates, and decrease the power to detect truly

209 differentially expressed genes (Robinson \& Oshlack, 2010). Before doing the differential

210 expression analyses and the estimation of tissue specificity, we evaluated the quality of

211 our samples by doing a principal component analysis (PCA) and a Pearson correlation

212 matrix using the gene raw count matrix, according to the Trinity QC samples and

213 biological replicates pipeline (https://github.com/trinityrnaseq/trinityrnaseq/wiki/QC-

214 Samples-and-Biological-Replicates). The intention of these analyses was to look for the

215 presence of batch effects or sample outliers, and to verify that biological replicates

216 clustered within each tissue type and not among sampled individuals.

217

218 Differential expression analysis and identification of tissue preferentially 219 upregulated genes.

220 Differentially expressed genes (DEG) and preferentially up-regulated genes (PUR) were

221 identified using the Trinity Differential Expression and Sample-Specific Expression

222 pipelines (Bryant et al., 2017, https://github.com/trinityrnaseq/trinityrnaseq/wiki/Trinity-

223 Differential-Expression). Briefly, we first identified DEG using the gene raw counts

224 matrix with edgeR 3.28.0 (Robinson, McCarthy \& Smyth, 2010; McCarthy, Chen \&

225 Smyth, 2012). The differential expression analysis was based on pairwise comparisons

226 of each of the 5 tissues, using the six samples per tissue as biological replicates, then

227 for each pair of DEG identified we obtained their associated false discovery rate (FDR).

228 Next, we obtained a normalized mean value of expression for each tissue by averaging

229 and $\log 2$ transforming the counts for each gene across the six replicates for each tissue

230 on the TMM gene matrix. Afterwards, pairwise comparisons of the averaged log2 counts

231 values per tissue were done and a logFC was assigned to each gene. DEG with a 
232 maximum FDR of 0.05 for differential expression, and with positive logFC in each

233 pairwise comparison of the averaged log2 TMM normalized counts was then classified

234 as PUR. A summary of pairwise expression differences between tissues based on the

$235 \log \mathrm{FC}$ of the log2 transformed gene counts in the TMM matrix is provided in Data S2

236 (https://figshare.com/articles/dataset/Data_S13_DE_pairwise_sumary_txt/13128476).

237

238 Tissue-specific expression

239 As an alternative approach to quantitatively assess the tissue-specific expression of the

240 genes we calculated the $\mathrm{T}$ index based on the TMM gene counts matrix. The $\mathrm{T}$ index

241 ranges between 0 for widely expressed genes, and 1 for exclusively tissue-specific

242 genes (Yanai et al., 2005) . As the $\mathrm{T}$ index considers tissue specificity independently of

243 the level of expression, we set as "not expressed" genes with expression values $<1$

244 from our TMM matrix in order to exclude genes with low support for true expression and

245 low signal to noise ratio. To do this, we first log2 transformed the matrix in order to

246 normalize the distribution of the expression values. We set all negative values in the

247 matrix to zero, as this represented values $<1$ before log2 transformation. We excluded

248 contigs that had no expression values or that had expression in just one out of the 30

249 samples. Then, the $\mathrm{T}$ index was computed separately for each gene across all tissues

250 and replicates following according to the following equation (Yanai et al., 2005;

251 Kryuchkova-Mostacci \& Robinson-Rechavi, 2017

252 https://github.com/severinEvo/gene_expression/blob/master/tau.R) :

253

254

$$
\tau=\frac{\sum_{i=1}^{N}\left(1-X_{i}\right)}{N-1}, X_{i}=\frac{x_{i}}{\max \left(x_{i}\right)} \text { where } \max \left(\mathrm{x}_{\mathrm{i}}\right) 1 \leq i \leq N
$$


256 Where $\mathrm{N}$ represents the number of tissues, $\mathrm{x}_{\mathrm{i}}$ is the mean expression in tissue $i$ and $\mathrm{X}_{\mathrm{i}}$

257 is the expression level in tissue $i$ normalized by the maximum mean expression among 258 all tissues.

\section{Singular enrichment analysis}

261 To further characterize the gene expression in the five tissues, we identified the

262 biological pathways for both tissue-specific and tissue preferentially upregulated gene

263 sets with independent singular enrichment analysis (SEA) (Huang, Sherman \&

264 Lempicki, 2009; Du et al., 2010). First, we retrieved the UniProt IDs corresponding to

265 our putative genes from the blastx field from our reference annotation file (Ojeda et al.,

266 2019). Then we uploaded the list of UniProt IDs to the uniprot retrieve/ID mapping tool

267 (https://www.uniprot.org/uploadlists/) and restricted the result to GO terms only. We

268 repeated this procedure with the genes used as a background list for the SEA: all the

269 contigs in the gene raw counts matrix for the PUR genes (Data S3 available at

270 https://figshare.com/articles/dataset/Data_S3_PUR_background_AgriGO/13553303),

271 and all the contigs in the filtered TMM matrix in the case of the tissue-specific genes

272 (Data S4 available at

273 https://figshare.com/articles/dataset/Data_S4_Tissue_specific_background_AgriGO/135

274 53306).

275 Of the 715,398 putative genes in the raw counts matrix used for the differential

276 expression analysis, 17,227 have a unique UniProt ID and represent 108,947 GO

277 terms. The background list for the tissue-specific genes data set consisted of 177,075 
278 contigs of which 14,079 have a unique annotation and represent $90,198 \mathrm{GO}$ terms. For

279 both data sets only uniquely annotated genes and their corresponding GO terms (Data

280 S5-S14 available at https://figshare.com/account/home\#/collections/5264255) were

281 used for running the singular enrichment analyses to avoid inflating the number of GO

282 terms falsely, and creating a bias in the analysis.

283 We used the GO terms along the UniProt IDs as input for the SEA using the 284 agriGO (http://systemsbiology.cau.edu.cn/agriGOv2/index.php) platform (Du et al., 285 2010; Tian et al., 2017). We used the custom background list option, applied a 286 hypergeometric test as statistical test method with a minimum of 5 mapping entries per 287 term, and Hochberg FDR as multi-test adjustment method with a significance level of 288 0.05. As the significant enrichment of child terms can inflate the enrichment significance 289 of parental terms, after the SEA we used REVIGO (Supek et al., 2011, $290 \mathrm{http}: / /$ revigo.irb.hr/) to reduce the redundancy of the GO terms and highlight the unique 291 and non-dispensable terms per tissue for both PUR and tissue-specific genes. We used 292 the list of enriched GO terms found in the SEA and their respective p-value as input, 293 selected the small output setting for redundancy, the whole UniProt as database, and 294 SimRel as measure of semantic similarity.

Results and discussion

Transcript quantification and abundance matrices construction

We mapped a total of $707,063,773$ trimmed and adapter removed reads from five different tissues (embryo, megagametophyte, needle, phloem, and vegetative bud) and six biological replicates (six different genotypes) per tissue type to $P$. sylvestris 
301 TRINITY guided transcriptome (Ojeda et al., 2019). On average 23,568,792 reads

302 originated from each tissue, ranging from $29,591,629$ reads for needle to $20,469,80$

303 reads for phloem. On average $76 \%$ of the reads per replicate were successfully mapped

304 to the reference (Table S5 available at

305 https://figshare.com/articles/dataset/Table_S5_Reads_and_percentage_of_mapped_re

306 ad_per_tissue/13553438). After mapping 1,307,500 contigs had aligned reads at the

307 isoform level. Of those, 120,040 contigs were removed from the downstream analyses

308 as they were identified as contaminants (Data S15 available at

309 https://figshare.com/articles/dataset/Data_S1_List_of_putative_contaminant_contigs_re

310 moved_from_quantification_files_txt/13121849). The final set consisted of $1,187,460$

311 contigs at isoform level and were used to construct raw counts and normalized matrices

312 at gene level for downstream analyses (see Materials and Methods section). The total

313 number of putative genes with expression signal in the gene level matrices was

314715,398, much higher than the number of annotated genes in any conifer (Nystedt et

315 al., 2013; Neale et al., 2014; Gonzalez-lbeas et al., 2016). This magnitude, albeit

316 probably an overestimate, is typical to transcriptome studies (Little et al., 2016). This is

317 likely a result of single genes being present in multiple fragments, isoforms split into

318 multiple genes, and different alleles originating from heterozygous material identified as

319 separate genes during assembly and classification as genes by Trinity (Ojeda et al.,

320 2019). However, part of the genes originate from gene families and since clustering

321 similar genes is possible in downstream analysis, we chose to err on the side of

322 potentially over splitting the genes rather than imperfectly clustering similar transcripts

323 as a single gene, as over clustering will inherently lead to loss of information. We 
324 believe that providing expression data with minimum clustering will be most versatile for

325 later use of the transcriptome and expression data in genome annotations and other 326 studies.

327

\section{Quality assessment of biological replicates}

329 As we used different genotypes as biological replicates, we first verified that the

330 replicates clustered by tissue type and not by genotype, and checked for the presence

331 of potential outliers in the dataset. We used the raw counts matrix data (Table S2

332 available at

333 https://figshare.com/articles/dataset/Table_S2_raw_counts_matrix_txt/13121858), a

334 principal component analysis (PCA) and a Pearson correlation to verify this. The PCA

335 separated the tissue samples into five distinct clusters without any overlap, indicating

336 that among-tissue variation is the main factor of among-sample variation (Figure 1).

337 Hence, our approach captures the differentiating gene expression profiles of the five

338 tissues. In the PCA, the seed-derived megagametophyte and embryo samples clustered

339 closest to each other, suggesting similarity in their gene expression profiles. Also

340 phloem and bud samples clustered close to each other, whereas needle samples

341 showed the most unique gene expression profile. In the hierarchical clustering analysis,

342 based on the correlations of gene expression profiles, the differences among tissues

343 are relatively shallow. But, similarly to the PCA, all replicates are clustered according to

344 their tissue type and not according to their genotypes, corroborating the PCA results

345 (Figure S1 available at 
346 https://figshare.com/articles/figure/Figure_S1_Pearson_correlation_matrix_expression_f 347 ive_tissues/13553744).

349 Tissue preferentially upregulated and tissue-specific gene expression

350 We defined a gene as tissue PUR when there was a significant log fold change in the

351 expression value compared to the other tissues. To identify tissue PUR we first did a

352 differential expression (DE) analysis. For this we included all the genes in the raw count 353 matrix (Table S2,

354 https://figshare.com/articles/dataset/Table_S2_raw_counts_matrix_txt/13121858). We

355 decided not to apply any minimum number of counts per gene as a filtering threshold to 356 run the analysis, as we later applied a $5 \%$ false discovery rate (FDR) threshold for the 357 identification of PUR genes. Out of the 715,398 genes initially included in the DE 358 analysis, 198,413 genes had a maximum 5\% FDR for differential expression and were 359 further included in the analysis to identify PUR genes. We identified a total of 48,001 360 genes with tissue preferential expression, and out of the five tissues needle has the 361 highest number of PUR genes (Table 1)

362 Quantification of tissue specificity allows a powerful statistical analysis of 363 correlation between tissue-specific expression and e.g. evolutionary rate or other 364 dependent or explanatory variables and factors. We identified the tissue specifically 365 expressed genes by calculating the $\mathrm{t}$ score per gene. The score ranges from zero to one, with a zero given to genes expressed in all tissues and one given to completely

367 tissue specific genes. For this analysis we retained a set of 177,075 genes (Table S6 368 available at 
369 https://figshare.com/articles/dataset/Table_S3_Filtered_TMM_Tau_final_matrix_txt/131

370 21864) after applying the filtering criteria described in Methods. We considered a gene

371 as tissue specifically expressed only if its $\mathrm{T}=1$. We identified a total of 3,899 genes with

372 a tissue-specific pattern of expression. Similarly, the PUR analysis results, needle has

373 the highest number of tissue-specific genes (Table 1). To obtain the annotation of the

374 genes identified as tissue PUR and tissue specific, we retrieved the corresponding

375 UniProtKB identifiers (Ojeda, 2020) from the Trinotate for the 715,398 putative genes in

376 the TMM count matrix, out of which 97,435 (14\%) had a Swiss-Prot (Bairoch \&

377 Apweiler, 2000) protein match based on BLASTX (Ojeda et al., 2019). Most of the

378 Swiss-Prot annotations (67\%) originated from Arabidopsis thaliana (65,214 genes).

379 Other common annotation sources were Nicotiana tabacum $(9,794 ; 10 \%)$ and Oryza

380 sativa (8,946; 9\%). Only 1663 genes (1.7\%) had an annotation to other Pinus species,

381 of which $177(10.6 \%)$ were hits to P. sylvestris, and 608 (36.5\%) genes had Swiss-Prot

382 annotation to Picea. Note that Swiss-Prot is a manually curated database that does not

383 currently have a comprehensive set of annotated gymnosperm proteins and therefore

384 the best matches are often obtained from the model plants such as $A$. thaliana. A

385 proportion of our putative genes share the same gene identifier (annotation) (Table 1).

386 This probably reflects the incomplete collapse of different isoforms in the assembled

387 transcriptome used as reference, or the presence of gene families (Wegrzyn et al.,

388 2014). Also, a high number of the genes identified as PUR or tissue specific lack

389 annotation altogether, which is not surprising as genes with higher tissue-specific

390 expression have less conserved sequences and are less likely to find orthologs among

391 other species (Lemos et al., 2005; Raherison et al., 2012). A summary of the 715,398 
392 genes indicating their normalized expression level (TMM), т score, tissue specificity

393 status, PUR status, and annotation can be found in the Supplementary information

394 (Table S7 available at

395 https://figshare.com/articles/dataset/Table_S4_CombinedTissueExpressionlnfo_txt/131 396 21867).

397 Cursory inspection of annotations of highly expressed tissue PUR and tissue398 specific genes are congruent with some of the already known functions of the tissues. These results confirm that our analyses capture biologically meaningful characteristics 400 of the tissues. For example in megagametophytes, enzymes related to seed storage 401 lipid mobilization and germination were upregulated and specifically expressed. 402 Similarly, in needles, several chlorophyll a-b binding proteins are upregulated. In 403 embryo, multiple ribosomal proteins and other proteins indicating active protein 404 synthesis were upregulated. In vegetative buds, expression of genes involved in 405 defense against insect attack, like (-)-alpha-pinene synthase and dirigent (Ralph et al., 406 2006) that take part in oleoresin synthesis, were highly expressed and specific to this 407 tissue. In phloem, the two genes annotated as metallothionein-like protein EMB30, an 408 aquaporin and a thioredoxin-like protein were highly expressed, similarly to Quercus 409 suber phellem (cork) where metallothionein reacts to oxidative stress (Mir et al., 2004) 410 or in Pinus taeda xylem where the same proteins were among the most highly expressed genes (Lorenz \& Dean, 2002).

413 with tissue-specific expression and embryo the lowest (Table 1). Except for two genes, 414 one in megagametophyte and one in needle, all the genes with tissue-specific 
415 expression were also among the PUR genes. However, as tissue specificity does not

416 require a high expression level, genes with $\mathrm{t}$ score equal to one are not necessarily the

417 most upregulated genes in their respective tissues. Comparison of our findings to other

418 studies is not straightforward as there are very few transcriptomic studies in $P$.

419 sylvestris. But in comparison to a previous study (Merino et al., 2016), where they focus

420 on the comparison between megagametophyte and embryo tissues at different

421 developmental stages, we identified less megagametophyte and embryo specifically

422 expressed genes. One of the reasons for this difference could be that the identification

423 of unique genes in Merino et al. (2016) was based only on the comparison between

424 embryo and megagametophyte tissues. As the identification of tissue specific genes is

425 contingent to the number of tissues used for the analysis, it is expected that the higher

426 the number of tissues used in the comparison, the lower the number of tissue specific

427 genes that will be identified. In contrast, we found a higher number of tissue specific

428 genes in embryo, bud, and needle compared to a previous study in conifers (Raherison

429 et al., 2012), where several tissue types were used. One notable difference between

430 this (Raherison et al., 2012) and ours was the higher number of tissue-specific genes

431 for megagametophyte found in P. glauca. (Raherison et al., 2012) found the highest

432 number of unique genes in the megagametophyte in comparison to other tissues

433 analyzed. The low number of megagametophyte specific genes identified in our study

434 could be due to the use of mature embryos as starting material as previous research

435 suggests that the number of unique transcripts in the megagametophyte varies during

436 the developmental stages of embryogenesis (Merino et al., 2016). 
438 microdissection in order to obtain the tissue samples (Cañas et al., 2017). Hence, some

439 of the "tissues" are a mix of tissue types. Needles, for example, include several tissues

440 (phloem among them) (Pongrac et al., 2019), and mature embryos contain the shoot

441 and root meristems as well as cotyledons (Singh, 1978). In contrast, the mature

442 megagametophyte is a quite uniform storage tissue consisting of cells packed with

443 starch protein and lipids (Simola, 1974; Vuosku et al., 2015). Another limitation of the

444 dataset is that it represents only one point in time and space, although gene expression

445 is a dynamic process and quantitative and qualitative variations exist over spatial and

446 temporal scales. Instead of sampling across several developmental stages or across a

447 spatial gradient our dataset represents a wider set of tissues, which increases the

448 power to identify tissue PUR and tissue specifically expressed genes. The added value

449 of the dataset lies in the unexpected functions and connections discovered among

450 biological pathways and genes with previously unidentified signals of tissue-specificity

451 or up-regulation.

452

453 Functional characterization of tissue preferentially upregulated and tissue454 specific genes

455 GO enrichment analysis allows the identification of gene functions enriched with certain

456 functional roles. The number of enriched functions was of the same magnitude across

457 tissue types, ranging from 253 to 452 for PUR genes and from 58 to 169 for tissue-

458 specific genes (Table S8-S17 available at

459 https://figshare.com/account/home\#/collections/5264255). The total number of GO

460 terms, the number of significant enriched terms, and the number of terms after the 
461 reduction of redundancy are shown in Table 2. Most of the genes (86\%) with expression

462 signals in our study lacked annotation from the Trinotate pipeline. Thus, they did not

463 contribute to functional analysis or GO enrichment results. A summary of the most

464 highly expressed genes per tissue, and the most enriched, non-redundant GO terms in

465 the biological processes category are shown in Figure 2. The complete lists of gene

466 identifiers and their corresponding GO terms per tissue and per each set of genes (Data

467 S5-S14, https://figshare.com/account/home\#/collections/5264255), along with tables

468 with the results of the SEA showing each GO terms, its p-value, and FDR (Table S8-

469 S17, https://figshare.com/account/home\#/collections/5264255), and lists with levels of

470 uniqueness or indispensability for each significantly enriched term in the five tissues

471 (Data S16 available at https://figshare.com/account/articles/14519139) are provided in

472 supplementary information.

473 In needles the significant GO terms reflected the exposure of trees to various

474 stresses and interactions with other organisms, whereas in embryos, buds and the

475 phloem the GO terms were mainly connected to different development-related

476 processes. In needles the enriched biological process GO terms among tissue-specific

477 genes were related to immune response (GO:0006955) as well as response to stress

478 (GO:0006950) and other organisms (GO:0051707) such as oomycetes (GO:0002229),

479 bacteria (GO:0042742) and fungi (GO:0009817). Moreover, terpene synthase activity

480 (GO:0010333), which may play a key role in the defense against herbivores (Achotegui-

481 Castells et al., 2013), was an enriched molecular function among tissue-specific genes

482 in needles, but also in embryos and vegetative buds. For example, reactive oxygen

483 species (ROS) related biological processes (GO:0006800 and GO:0042743, 
484 GO:0034614) and molecular functions (GO:0004601, GO:0004364) were enriched 485 among the GO terms in the tissue-specific genes of embryos, which is consistent with 486 an active ROS protection in developing tissues. In the phloem, a special differentiation 487 process, syncytium formation (GO:0006949), indicating the interconnection of phloem 488 sieve elements to generate a transport route (Geldner, 2014) was an enriched biological 489 process among the tissue specific genes.

490

491

492

493

494

495

496

497

498

499

500

501

502

503

504

505

506

Megagametophyte-specific genes have crucial functions in seed germination and energy conversion

Gymnosperms are characterized by the haploid female gametophyte tissue, the megagametophyte, which surrounds the embryo in developing and mature seeds. The megagametophyte can be considered a functional homolog of the endosperm in angiosperms due to its role as a nourishing tissue (King \& Gifford, 1997; Costa, Gutièrrez-Marcos \& Dickinson, 2004). However, the megagametophyte develops from a haploid megaspore before the fertilization (Singh, 1978) and is therefore entirely maternally inherited unlike the diploid or triploid endosperms of biparental origin (Williams \& Friedman, 2002; Baroux, Spillane \& Grossniklaus, 2002). To give an example of the potential uses of the dataset, we provide a more detailed description of the megagametophyte expression profile, but leave the in-depth analysis of the other tissues for later investigations.

Among highly expressed and up-regulated genes in the megagametophyte were malate synthase (EC 2.3.3.9) and isocitrate lyase (EC 4.1.3.1) that are essential in glyoxylate cycle converting lipids into carbohydrates in seeds (Ching, 1970), as well as 
507 other glyoxysomal proteins like Acetyl-CoA acyltransferase (EC 2.3.1.16), ABC

508 transporter and peroxisomal fatty acid beta-oxidation multifunctional protein AIM1

509 (Graham, 2008). Seed storage related genes such as 2S seed storage-like protein, 11S

510 globulin seed storage protein 2 and $13 S$ globulin basic chain and some isocitrate lyase

511 copies were completely megagametophyte specific $(\mathrm{T}=1)$. Antimicrobial and antifungal

512 protein coding genes were the most highly expressed among annotated

513 megagametophyte-upregulated genes.

514 The enriched GO terms of biological processes and molecular functions in the

515 megagametophyte tissue-specific genes included seed germination and the mobilization

516 of nutrient reserves. Nutrient reservoir activity (GO:0045735) indicated the mobilization

517 of energy sources from the megagametophyte for seed germination and early seedling

518 growth, as well as lipid catabolic processes (e.g. GO:0016042, GO:0044242). Malate

519 dehydrogenase activity (GO:0016615) and heme binding (GO:0020037), which mostly

520 originated from the cytochrome P450 enzymes containing heme cofactors ( $\mathrm{Xu}$ et al.,

521 2015), reflected the resume of active metabolism. Also, response to ROS

522 (GO:0034614) and antioxidant activity (GO:0016209) suggested active metabolism and

523 signaling. ROS are natural by-products of metabolism and may be detrimental to seed

524 viability because they can cause oxidative stress. However, in the seed ROS also work

525 as signals which underpin the breaking of dormancy and provide protection against

526 pathogens (Jeevan Kumar et al., 2015). Megagametophyte cells showed responses to

527 hormone stimulus (GO:0032870) and the function of hormone-mediated signaling

528 pathways (GO:0009755) including abscisic acid (GO:0009738), auxin (GO:0009734)

529 and ethylene (GO:0009873) which also belong to the molecular networks regulating 
530 seed dormancy and germination (Seo et al., 2009; Guangwu \& Xuwen, 2014; Miransari

531 \& Smith, 2014; Shu et al., 2016). Cellulose biosynthetic process (GO:0030244) and

532 primary cell wall biogenesis (GO:0009833) suggest that cell walls in the

533 megagametophyte may participate in water retention and give mechanical support to

534 the germinating embryo (Otegui, 2007). Similarly to previous findings in $P$. sylvestris

535 (Merino et al., 2016) megagametophytes, we found enrichment for processes involved

536 in the response to chemical and endogenous stimuli (GO:0042221, GO:0071495).

537 Merino et al. (2016) suggested that the megagametophyte could also be involved in the

538 regulation of the embryo development through the induction of signaling pathways

539 triggered by sensing environmental signals in a similar way the angiosperms'

540 endosperm does (Yan et al., 2014). Altogether, our findings show that the

541 megagametophyte is not just a reserve nutrition for the germinating embryo, but a

542 metabolically active tissue contributing in multiple ways to seed germination and, thus,

543 underline the importance of the haploid stage in $P$. sylvestris life cycle.

$544 \quad$ Several enzymes widely used in allozyme-based population genetic studies

545 ((Szmidt \& Muona, 1989) and references therein) such as aconitate hydratase (EC

546 4.2.1.3), malate dehydrogenase (EC 1.1.1.37) and aspartate aminotransferase (EC

547 2.6.1.1) were megagametophyte-specific and among the top 50 expressed genes in the

548 tissue. As they may be more prone to natural selection against recessive deleterious

549 variants when expressed at the haploid stage, early population genetic analyses may

550 have bias in e.g. estimates of the overall genetic diversity based on these loci as highly

551 expressed genes are known to be under strong purifying selection. 


\section{Conclusions}

553 We provide a widely and interdisciplinary applicable genome-wide atlas of tissue-level

554 transcription patterns based on RNA-seq for economically and ecologically significant

555 coniferous tree $P$. sylvestris. Quantitative data and analysis of expression level, as well

556 as breadth and tissue specificity are provided for 715,398 different putative genes. The

557 mapping and bioinformatic analyses of gene expression are based on the most

558 complete and high-quality reference transcriptome of $P$. sylvestris available to date

559 (Ojeda et al., 2019). Previous transcriptome studies of $P$. sylvestris have concentrated

560 on a narrow set of tissues in each study such as wood (Paasela et al., 2017), embryo

561 (Merino et al., 2016), and needles (Wachowiak et al., 2015; Duarte, Volkova \&

562 Geras'kin, 2019) or focused on a limited set of genes (Guseva, Biriukov \& Sadovsky,

563 2020). The present study allows comparison across a wide set of genes expressed in

564 the above-ground parts of adult $P$. sylvestris trees growing in a natural forest.

565 In addition to genome sequence annotations, we foresee multiple potential uses

566 for the dataset. Level and breadth of gene expression are known to be linked to the

567 evolutionary rate and level of conservation (Lemos et al. 2005, Brawand et al. 2011,

568 Yang and Wang 2013). By combining our data with similar data in other conifers or

569 angiosperms it is possible to study the evolutionary conservation of expression patterns,

570 or the differences in evolutionary rates across tissue-specific expression levels and gain

571 a deeper understanding of the determinants and main factors affecting e.g. rate of

572 adaptive evolution and dynamics at the genome level. The response of trees to a

573 combination of different stresses is unique and cannot be directly extrapolated from

574 studying only single stressors in experimental conditions (Niinemets, 2010). The

575 transcriptome resource for adult $P$. sylvestris trees growing under natural conditions, 
576 where they are simultaneously exposed to a number of different abiotic and biotic

577 stresses as well as interactions with other organisms, provides a valuable tool also for

578 physiological studies. Finally, un-annotated conifer genes with high expression or tissue

579 specificity can open up whole new research avenues, independent of the previously

580 available knowledge based on angiosperm model plants such as $A$. thaliana and

581 Populus.

582

\section{Supplementary information}

584 All the supplementary files can be found at https://figshare.com/s/baae929be89134ad2dcd

585

586

\section{References}

587 Achotegui-Castells A, Llusià J, Hódar JA, Peñuelas J. 2013. Needle terpene concentrations and 588 emissions of two coexisting subspecies of Scots pine attacked by the pine processionary 589

590

591

592

593

594

595

596

597

598 moth (Thaumetopoea pityocampa). Acta Physiologiae Plantarum 35:3047-3058. DOI: 10.1007/s11738-013-1337-3.

Bairoch A, Apweiler R. 2000. The SWISS-PROT protein sequence database and its supplement TrEMBL in 2000. Nucleic acids research 28:45-48.

Baker EAG, Wegrzyn JL, Sezen UU, Falk T, Maloney PE, Vogler DR, Delfino-Mix A, Jensen C, Mitton J, Wright J, Knaus B, Rai H, Cronn R, Gonzalez-lbeas D, Vasquez-Gross HA, Famula RA, Liu J-J, Kueppers LM, Neale DB. 2018. Comparative Transcriptomics Among Four White Pine Species. G3 8:1461-1474.

Baroux C, Spillane C, Grossniklaus U. 2002. Evolutionary origins of the endosperm in flowering plants. Genome biology 3:1-5. 
599 Birol I, Raymond A, Jackman SD, Pleasance S, Coope R, Taylor GA, Yuen MMS, Keeling Cl, 600 Brand D, Vandervalk BP, Kirk H, Pandoh P, Moore RA, Zhao Y, Mungall AJ, Jaquish B, 601 Yanchuk A, Ritland C, Boyle B, Bousquet J, Ritland K, Mackay J, Bohlmann J, Jones SJM. 602 2013. Assembling the $20 \mathrm{~Gb}$ white spruce (Picea glauca) genome from whole-genome 603 shotgun sequencing data. Bioinformatics 29:1492-1497.

604 Bonan GB, Chapin FS, Thompson SL. 1995. Boreal forest and tundra ecosystems as 605 components of the climate system. Climatic change 29:145-167.

606 Boonstra R, Andreassen HP, Boutin S, Hušek J, Ims RA, Krebs CJ, Skarpe C, Wabakken P. 607 2016. Why Do the Boreal Forest Ecosystems of Northwestern Europe Differ from Those of 608 Western North America? Bioscience 66:722-734.

609 Brawand D, Soumillon M, Necsulea A, Julien P, Csárdi G, Harrigan P, Weier M, Liechti A, 610 Aximu-Petri A, Kircher M, Albert FW, Zeller U, Khaitovich P, Grützner F, Bergmann S, 611 Nielsen R, Pääbo S, Kaessmann H. 2011. The evolution of gene expression levels in 612 mammalian organs. Nature 478:343-348.

613 Cañas RA, Li Z, Pascual MB, Castro-Rodríguez V, Ávila C, Sterck L, Van de Peer Y, Cánovas 614 FM. 2017. The gene expression landscape of pine seedling tissues. The Plant journal: for cell and molecular biology 91:1064-1087.

Cañas RA, Pascual MB, Fernando N, Ávila C, Cánovas FM. 2019. Resources for conifer functional genomics at the omics era. In: Advances in Botanical Research. Elsevier, 39-76.

Ching TM. 1970. Glyoxysomes in megagamethophyte of germinating ponderosa pine seeds. Plant physiology 46:475-482.

621 complex times of the plant endosperm. Trends in plant science 9:507-514.

622 DeAngelis DL. 2008. Boreal Forest. Encyclopedia of Ecology:493-495. DOI: 10.1016/b978$623 \quad 008045405-4.00319-0$.

624 De La Torre AR, Piot A, Liu B, Wilhite B, Weiss M, Porth I. 2020. Functional and morphological 
625 evolution in gymnosperms: A portrait of implicated gene families. Evolutionary applications $626 \quad 13: 210-227$.

627 Duarte GT, Volkova PY, Geras'kin SA. 2019. The response profile to chronic radiation exposure 628 based on the transcriptome analysis of Scots pine from Chernobyl affected zone.

629 Environmental pollution 250:618-626.

630 Du Z, Zhou X, Ling Y, Zhang Z, Su Z. 2010. agriGO: a GO analysis toolkit for the agricultural 631 community. Nucleic acids research 38:W64-70.

632 FAO Yearbook of Forest Products. 2020. Food and Agriculture Organization of the United $633 \quad$ Nations.

634 Farjon A. 2008. A natural history of conifers.

635 Geldner N. 2014. Making phloem--a near-death experience. Science 345:875-876. DOI:

$636 \quad 10.1126 /$ science. 1258711.

637 Gonzalez-Ibeas D, Martinez-Garcia PJ, Famula RA, Delfino-Mix A, Stevens KA, Loopstra CA, 638 Langley $\mathrm{CH}$, Neale DB, Wegrzyn JL. 2016. Assessing the Gene Content of the 639 Megagenome: Sugar Pine (Pinus lambertiana). G3 6:3787-3802.

640 Graham IA. 2008. Seed Storage Oil Mobilization. Annual Review of Plant Biology 59:115-142.

641 DOI: 10.1146/annurev.arplant.59.032607.092938.

642 Guangwu Z, Xuwen J. 2014. Roles of Gibberellin and Auxin in Promoting Seed Germination and 643 Seedling Vigor in Pinus massoniana. Forest Science 60:367-373. DOI: 10.5849/forsci.12644143.

645 Guseva T, Biriukov V, Sadovsky M. 2020. Role of Homeobox Genes in the Development of 646 Pinus Sylvestris. Bioinformatics and Biomedical Engineering:429-437. DOI: 10.1007/978-3647 030-45385-5_38.

648 Höllbacher B, Schmitt AO, Hofer H, Ferreira F, Lackner P. 2017. Identification of Proteases and 649 Protease Inhibitors in Allergenic and Non-Allergenic Pollen. International journal of 650 molecular sciences 18. DOI: 10.3390/ijms18061199. 
651 Huang DW, Sherman BT, Lempicki RA. 2009. Bioinformatics enrichment tools: paths toward the 652 comprehensive functional analysis of large gene lists. Nucleic acids research 37:1-13.

653 Jeevan Kumar SP, Rajendra Prasad S, Banerjee R, Thammineni C. 2015. Seed birth to death: 654 dual functions of reactive oxygen species in seed physiology. Annals of botany 116:663$655 \quad 668$.

656 King JE, Gifford DJ. 1997. Amino Acid Utilization in Seeds of Loblolly Pine during Germination 657 and Early Seedling Growth (I. Arginine and Arginase Activity). Plant physiology 113:1125$658 \quad 1135$.

659 Kryuchkova-Mostacci N, Robinson-Rechavi M. 2017. A benchmark of gene expression tissue660 specificity metrics. Briefings in bioinformatics 18:205-214.

661 Lemos B, Meiklejohn CD, Cáceres M, Hartl DL. 2005. Rates of divergence in gene expression 662 profiles of primates, mice, and flies: stabilizing selection and variability among functional 663 categories. Evolution; international journal of organic evolution 59:126-137.

664 Li Z, De La Torre AR, Sterck L, Cánovas FM, Avila C, Merino I, Cabezas JA, Cervera MT, 665 Ingvarsson PK, Van de Peer Y. 2017. Single-Copy Genes as Molecular Markers for 666 Phylogenomic Studies in Seed Plants. Genome biology and evolution 9:1130-1147.

667 Little SA, Boyes IG, Donaleshen K, von Aderkas P, Ehlting J. 2016. A transcriptomic resource 668 for Douglas-fir seed development and analysis of transcription during late 669 megagametophyte development. Plant reproduction 29:273-286.

670 Lorenz WW, Dean JFD. 2002. SAGE profiling and demonstration of differential gene expression 671 along the axial developmental gradient of lignifying xylem in loblolly pine (Pinus taeda). 672 Tree physiology 22:301-310.

673 McCarthy DJ, Chen Y, Smyth GK. 2012. Differential expression analysis of multifactor RNA-Seq 674 experiments with respect to biological variation. Nucleic acids research 40:4288-4297.

675 Merino I, Abrahamsson M, Sterck L, Craven-Bartle B, Canovas F, von Arnold S. 2016.

676 Transcript profiling for early stages during embryo development in Scots pine. BMC plant 
biology 16:255.

678 Miransari M, Smith DL. 2014. Plant hormones and seed germination. Environmental and

679 Experimental Botany 99:110-121. DOI: 10.1016/j.envexpbot.2013.11.005.

680 Mir G, Domènech J, Huguet G, Guo W-J, Goldsbrough P, Atrian S, Molinas M. 2004. A plant

681 type 2 metallothionein (MT) from cork tissue responds to oxidative stress. Journal of 682 experimental botany 55:2483-2493.

683 Mosca E, Cruz F, Gómez-Garrido J, Bianco L, Rellstab C, Brodbeck S, Csilléry K, Fady B, 684 Fladung M, Fussi B, Gömöry D, González-Martínez SC, Grivet D, Gut M, Hansen OK, Heer 685 K, Kaya Z, Krutovsky KV, Kersten B, Liepelt S, Opgenoorth L, Sperisen C, Ullrich KK, 686 Vendramin GG, Westergren M, Ziegenhagen B, Alioto T, Gugerli F, Heinze B, Höhn M, 687 Troggio M, Neale DB. 2019. A Reference Genome Sequence for the European Silver Fir (Abies alba Mill.): A Community-Generated Genomic Resource. G3 9:2039-2049.

Neale DB, Wegrzyn JL, Stevens KA, Zimin AV, Puiu D, Crepeau MW, Cardeno C, Koriabine M, Holtz-Morris AE, Liechty JD, Martínez-García PJ, Vasquez-Gross HA, Lin BY, Zieve JJ,

691

692

693

694

695

696 697 from seedlings to mature plants: Past stress history, stress interactions, tolerance and 698 acclimation. Forest Ecology and Management 260:1623-1639. DOI:

$699 \quad$ 10.1016/j.foreco.2010.07.054. Dougherty WM, Fuentes-Soriano S, Wu L-S, Gilbert D, Marçais G, Roberts M, Holt C,

2
Yandell M, Davis JM, Smith KE, Dean JFD, Lorenz WW, Whetten RW, Sederoff R, Wheeler

N, McGuire PE, Main D, Loopstra CA, Mockaitis K, deJong PJ, Yorke JA, Salzberg SL,

694

Langley CH. 2014. Decoding the massive genome of loblolly pine using haploid DNA and 595 novel assembly strategies. Genome biology 15:R59.

Niinemets Ü. 2010. Responses of forest trees to single and multiple environmental stresses

700 Nystedt B, Street NR, Wetterbom A, Zuccolo A, Lin Y-C, Scofield DG, Vezzi F, Delhomme N, 701 Giacomello S, Alexeyenko A, Vicedomini R, Sahlin K, Sherwood E, Elfstrand M, Gramzow 702 L, Holmberg K, Hällman J, Keech O, Klasson L, Koriabine M, Kucukoglu M, Käller M, 
703

704

705

706

707

708

709

710

711

712

713

714

715

716

717

718

719

720

721

722

723

724

725

726

727

728

Luthman J, Lysholm F, Niittylä T, Olson A, Rilakovic N, Ritland C, Rosselló JA, Sena J, Svensson T, Talavera-López C, Theißen G, Tuominen H, Vanneste K, Wu Z-Q, Zhang B, Zerbe P, Arvestad L, Bhalerao R, Bohlmann J, Bousquet J, Garcia Gil R, Hvidsten TR, de Jong P, MacKay J, Morgante M, Ritland K, Sundberg B, Thompson SL, Van de Peer Y, Andersson B, Nilsson O, Ingvarsson PK, Lundeberg J, Jansson S. 2013. The Norway spruce genome sequence and conifer genome evolution. Nature 497:579-584.

Ojeda D. 2020. Pinus sylvestris transcriptome annotation-Trinotate. Utilization of Tissue Ploidy Level Variation in de Novo Transcriptome Assembly of Pinus sylvestris. DOI: 10.6084/m9.figshare.12865997.v1.

Ojeda DI, Mattila TM, Ruttink T, Kujala ST, Kärkkäinen K, Verta J-P, Pyhäjärvi T. 2019. Utilization of Tissue Ploidy Level Variation in de Novo Transcriptome Assembly of Pinus sylvestris. G3 9:3409-3421.

Otegui MS. 2007. Endosperm Cell Walls: Formation, Composition, and Functions. In: Olsen O-A ed. Endosperm: Developmental and Molecular Biology. Berlin, Heidelberg: Springer Berlin Heidelberg, 159-177.

Otto SP, Scott MF, Immler S. 2015. Evolution of haploid selection in predominantly diploid organisms. Proceedings of the National Academy of Sciences of the United States of America 112:15952-15957.

Paasela T, Lim K-J, Pietiäinen M, Teeri TH. 2017. The O-methyltransferase PMT2 mediates methylation of pinosylvin in Scots pine. The New phytologist 214:1537-1550.

Patro R, Duggal G, Love MI, Irizarry RA, Kingsford C. 2017. Salmon provides fast and biasaware quantification of transcript expression. Nature methods 14:417-419.

Perry A, Wachowiak W, Downing A, Talbot R, Cavers S. 2020. Development of a SNP array for population genomic studies in four European pine species. Molecular ecology resources. DOI: $10.1111 / 1755-0998.13223$.

Pongrac P, Baltrenaite E, Vavpetič P, Kelemen M, Kladnik A, Budič B, Vogel-Mikuš K, Regvar

Peer] reviewing PDF | (2021:01:57009:1:1:NEW 11 May 2021) 
729

730

731

732

733

734

735

736

737

738

739

740

741

742

743

744

745

746

747

748

749

750

751

752

753

754

M, Baltrenas P, Pelicon P. 2019. Tissue-specific element profiles in Scots pine (Pinus sylvestris L.) needles. Trees 33:91-101.

Pyhäjärvi T, Kujala ST, Savolainen O. 2020. 275 years of forestry meets genomics in. Evolutionary applications 13:11-30.

Raherison E, Rigault P, Caron S, Poulin P-L, Boyle B, Verta J-P, Giguère I, Bomal C, Bohlmann J, MacKay J. 2012. Transcriptome profiling in conifers and the PiceaGenExpress database show patterns of diversification within gene families and interspecific conservation in vascular gene expression. BMC genomics 13:434.

Ralph S, Park J-Y, Bohlmann J, Mansfield SD. 2006. Dirigent proteins in conifer defense: gene discovery, phylogeny, and differential wound- and insect-induced expression of a family of DIR and DIR-like genes in spruce (Picea spp.). Plant molecular biology 60:21-40.

Rellstab C, Dauphin B, Zoller S, Brodbeck S, Gugerli F. 2019. Using transcriptome sequencing and pooled exome capture to study local adaptation in the giga-genome of Pinus cembra. Molecular ecology resources 19:536-551.

Robinson MD, McCarthy DJ, Smyth GK. 2010. edgeR: a Bioconductor package for differential expression analysis of digital gene expression data. Bioinformatics 26:139-140.

Robinson MD, Oshlack A. 2010. A scaling normalization method for differential expression analysis of RNA-seq data. Genome biology 11:R25.

San-Miguel-Ayanz J, De Rigo D, Caudullo G, Durrant TH, Mauri A. 2016. European atlas of forest tree species. Publications Office of the European Union.

Seo M, Nambara E, Choi G, Yamaguchi S. 2009. Interaction of light and hormone signals in germinating seeds. Plant molecular biology 69:463-472.

Shu K, Liu X-D, Xie Q, He Z-H. 2016. Two Faces of One Seed: Hormonal Regulation of Dormancy and Germination. Molecular plant 9:34-45.

Simola LK. 1974. The Ultrastructure of Dry and Germinating Seeds of Pinus Sylvestris L. Societas pro Fauna et Flora Fennica.

Peer] reviewing PDF | (2021:01:57009:1:1:NEW 11 May 2021) 
755 Singh H. 1978. Embryology of Gymnosperms.

756 Slotte T, Bataillon T, Hansen TT, St Onge K, Wright SI, Schierup MH. 2011. Genomic

757 determinants of protein evolution and polymorphism in Arabidopsis. Genome biology and

758 evolution 3:1210-1219.

759 Soneson C, Love MI, Robinson MD. 2015. Differential analyses for RNA-seq: transcript-level

760 estimates improve gene-level inferences. F1000Research 4:1521.

761 Stevens KA, Wegrzyn JL, Zimin A, Puiu D, Crepeau M, Cardeno C, Paul R, Gonzalez-lbeas D,

762 Koriabine M, Holtz-Morris AE, Martínez-García PJ, Sezen UU, Marçais G, Jermstad K,

763 McGuire PE, Loopstra CA, Davis JM, Eckert A, de Jong P, Yorke JA, Salzberg SL, Neale

764 DB, Langley CH. 2016. Sequence of the Sugar Pine Megagenome. Genetics 204:1613-

$765 \quad 1626$.

766 Supek F, Bošnjak M, Škunca N, Šmuc T. 2011. REVIGO summarizes and visualizes long lists

767 of gene ontology terms. PloS one 6:e21800.

768 Szmidt AE, Muona O. 1989. Linkage relationships of allozyme loci in Pinus sylvestris. Hereditas

769 111:91-97. DOI: 10.1111/j.1601-5223.1989.tb00382.x.

770 Tian T, Liu Y, Yan H, You Q, Yi X, Du Z, Xu W, Su Z. 2017. agriGO v2.0: a GO analysis toolkit

771 for the agricultural community, 2017 update. Nucleic acids research 45:W122-W129.

772 Tyrmi JS, Vuosku J, Acosta JJ, Li Z, Sterck L, Cervera MT, Savolainen O, Pyhäjärvi T. 2020.

773 Genomics of Clinal Local Adaptation in Pinus sylvestris Under Continuous Environmental

774 and Spatial Genetic Setting. G3: Genes|Genomes|Genetics 10:2683-2696. DOI:

$775 \quad 10.1534 / g 3.120 .401285$.

776 Verta JP, Landry CR, MacKay J. 2016. Dissection of expression-quantitative trait locus and

777 allele specificity using a haploid/diploid plant system-insights into compensatory evolution 778 of transcriptional .... The New phytologist.

779 Vuosku J, Sutela S, Kestilä J, Jokela A, Sarjala T, Häggman H. 2015. Expression of catalase 780 and retinoblastoma-related protein genes associates with cell death processes in Scots 
781 pine zygotic embryogenesis. BMC plant biology 15:88.

782 Wachowiak W, Trivedi U, Perry A, Cavers S. 2015. Comparative transcriptomics of a complex of 783 four European pine species. BMC genomics 16:234.

784 Wegrzyn JL, Falk T, Grau E, Buehler S, Ramnath R, Herndon N. 2020. Cyberinfrastructure and 785 resources to enable an integrative approach to studying forest trees. Evolutionary 786 applications 13:228-241.

787 Wegrzyn JL, Liechty JD, Stevens KA, Wu L-S, Loopstra CA, Vasquez-Gross HA, Dougherty 788 WM, Lin BY, Zieve JJ, Martínez-García PJ, Holt C, Yandell M, Zimin AV, Yorke JA, 789 Crepeau MW, Puiu D, Salzberg SL, Dejong PJ, Mockaitis K, Main D, Langley CH, Neale 790 DB. 2014. Unique features of the loblolly pine (Pinus taeda L.) megagenome revealed 791 through sequence annotation. Genetics 196:891-909.

792 Williams CG. 2008. Selfed embryo death in Pinus taeda: a phenotypic profile. The New 793 phytologist 178:210-222.

794 Williams JH, Friedman WE. 2002. Identification of diploid endosperm in an early angiosperm 795 lineage. Nature 415:522-526. DOI: 10.1038/415522a.

796 Wright SI, Yau CBK, Looseley M, Meyers BC. 2004. Effects of gene expression on molecular 797 evolution in Arabidopsis thaliana and Arabidopsis lyrata. Molecular biology and evolution $798 \quad 21: 1719-1726$.

799 Xu J, Jun XU, Wang X-Y, Wang-zhen GUO. 2015. The cytochrome P450 superfamily: Key 800 players in plant development and defense. Journal of Integrative Agriculture 14:1673-1686. 801 DOI: 10.1016/s2095-3119(14)60980-1.

802 Yanai I, Benjamin H, Shmoish M, Chalifa-Caspi V, Shklar M, Ophir R, Bar-Even A, Horn-Saban 803 S, Safran M, Domany E, Lancet D, Shmueli O. 2005. Genome-wide midrange transcription 804 profiles reveal expression level relationships in human tissue specification. Bioinformatics $805 \quad 21: 650-659$.

806 Yan D, Duermeyer L, Leoveanu C, Nambara E. 2014. The functions of the endosperm during 
807 seed germination. Plant \& cell physiology 55:1521-1533.

808 Yang R, Wang X. 2013. Organ evolution in angiosperms driven by correlated divergences of 809 gene sequences and expression patterns. The Plant cell 25:71-82.

810 Zimin A, Stevens KA, Crepeau MW, Holtz-Morris A, Koriabine M, Marçais G, Puiu D, Roberts M, 811 Wegrzyn JL, de Jong PJ, Neale DB, Salzberg SL, Yorke JA, Langley CH. 2014.

812 Sequencing and assembly of the 22-gb loblolly pine genome. Genetics 196:875-890.

813 Zonneveld BJM. 2012. Conifer genome sizes of 172 species, covering 64 of 67 genera, range 814 from 8 to 72 picogram. Nordic journal of botany 30:490-502.

815

816

\section{List of figures}

818 Figure 1 A) Schematic representation of the five tissues used in the transcriptome 819 profiling of Pinus sylvestris: needle, vegetative bud, megagametophyte, embryo and 820 phloem. B) Scatterplot of the first two axes of the principal component analysis (PCA).

821 Tissue types are denoted by colors. Figure draw and designed by Dorota Paczesniak

822 Figure 2 Ten most significant non-redundant biological processes and their

823 corresponding GO-term IDs (terms chosen based on the lowest dispensability value),

824 and ten most highly expressed annotated genes in each of the five tissues. Genes

825 preferentially upregulated (PUR) in a given tissue are in bold. Figure draw and designed 826 by Dorota Paczesniak. 
Figure 1

Schematic representation of the five tissues used and a scatterplot of the first two axes of the principal component analysis (PCA) of expression.

(A) Schematic representation of the five tissues used in the transcriptome profiling of Pinus sylvestris : needle, vegetative bud, megagametophyte, embryo and phloem. (B) Scatterplot of the first two axes of the principal component analysis (PCA). Tissue types are denoted by colors. Illustrations by Dorota Paczesniak.

A.

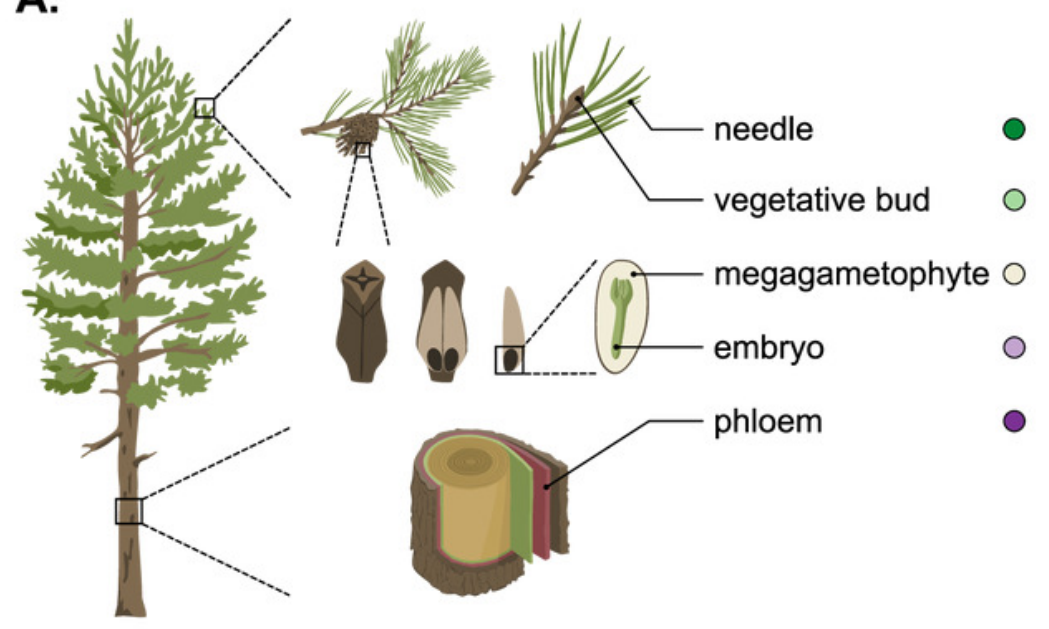

B.

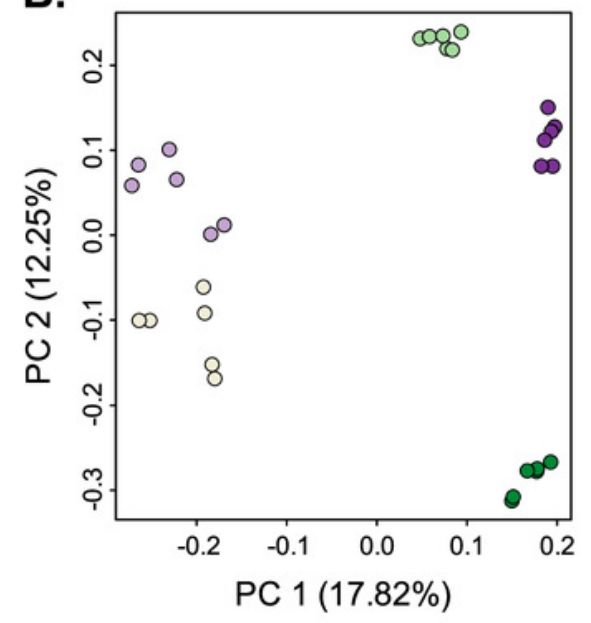




\section{Table 1 (on next page)}

Genes identified as preferentially upregulated and tissue specific

Table 1. Number of genes identified as tissue preferentially upregulated and tissue-specific in five $P$. sylvestris tissues. The percentage of unique UniProtKB identifiers is also shown. 
1 Table 1. Number of genes identified as tissue preferentially upregulated and tissue-

2 specific in five $P$. sylvestris tissues. The percentage of unique UniProtKB identifiers is

3 also shown.

\begin{tabular}{|l|l|l|l|l|l|l|}
\hline \multirow{2}{*}{} & \multicolumn{3}{|l|}{$\begin{array}{l}\text { Tissue preferentially upregulated } \\
\text { genes }\end{array}$} & \multicolumn{2}{l|}{ Tissue specifically expressed genes } \\
\cline { 2 - 7 } & Total & Annotated & Unique (\%) & Total & Annotated & Unique (\%) \\
\hline Bud & 8225 & 2515 & 30.6 & 693 & 342 & 49.3 \\
\hline Embryo & 10430 & 2820 & 27.0 & 498 & 206 & 41.3 \\
\hline Megagametophyte & 7171 & 1515 & 21.1 & 679 & 220 & 32.4 \\
\hline Needle & 13128 & 3993 & 30.4 & 1495 & 603 & 40.3 \\
\hline Phloem & 9047 & 2603 & 28.7 & 534 & 202 & 37.8 \\
\hline
\end{tabular}

4 


\section{Figure 2}

Ten most significant non-redundant biological processes

Ten most significant non-redundant biological processes and their corresponding GO-term IDs (terms chosen based on the lowest dispensability value), and ten most highly expressed annotated genes in each of the five tissues. Genes preferentially upregulated (PUR) in a given tissue are in bold. Illustration and design by Dorota Paczesniak 

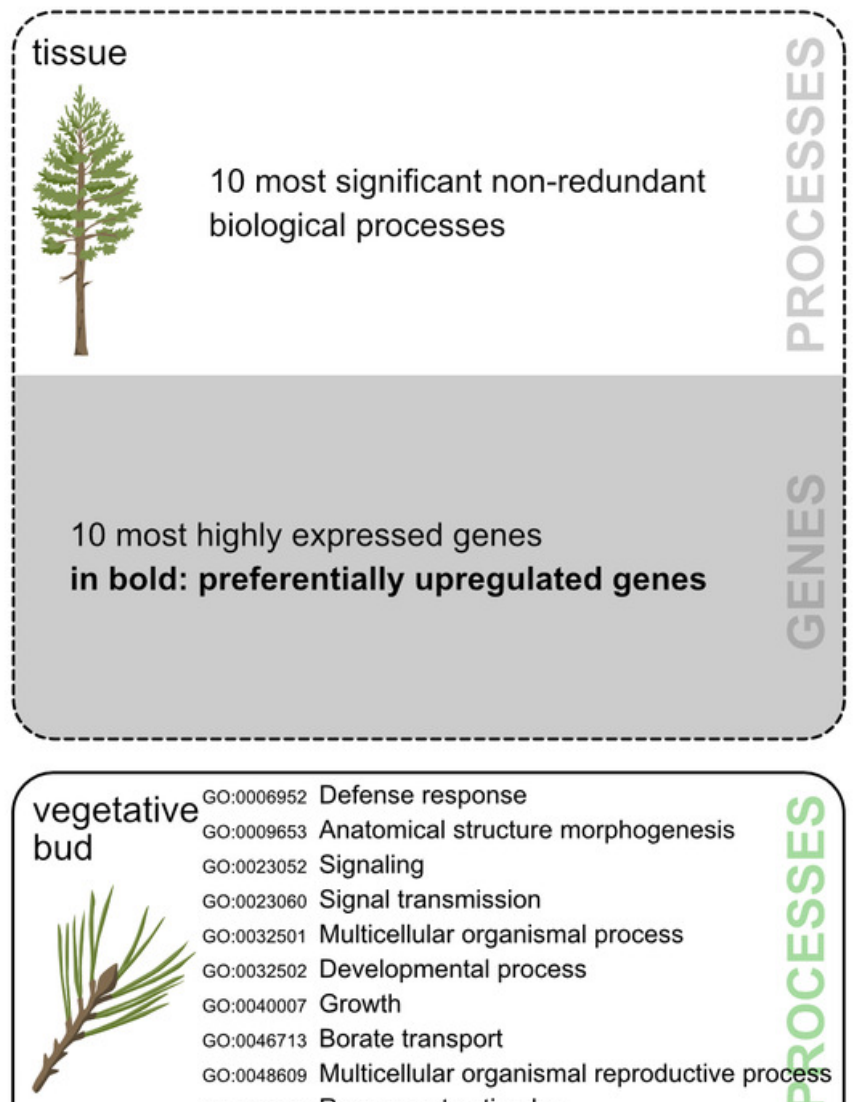

GO:0006952 Defense response

GO:0009653 Anatomical structure morphogenesis

GO:0023052 Signaling

GO:0023060 Signal transmission

GO:0032501 Multicellular organismal process

Go:0032502 Developmental process

Go:0040007 Growth

GO:0046713 Borate transport

Go:0048609 Multicellular organismal reproductive process

GO:0050896 Response to stimulus

Glycine-rich RNA-binding protein GRP1A

Chlorophyll a-b binding protein 16 , chloroplastic

Metallothionein-like protein EMB30

Abscisic stress-ripening protein 1

Cold shock domain-containing protein 4

Cysteine proteinase 15A

Major pollen allergen Bet $v$ 1-A

S-adenosylmethionine decarboxylase proenzyme

Non-specific lipid-transfer protein

High mobility group B protein 2 megagametophyte

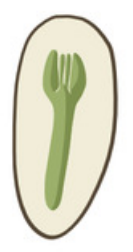

Go:0006097 Glyoxylate cycle

Go:0006732 Coenzyme metabolic process

Go:0006800 Oxygen and ROS metabolic process

GO:0009109 Coenzyme catabolic process

Go:0010087 Phloem and xylem histogenesis

GO:0042542 Response to hydrogen peroxide

GO:0051186 Cofactor metabolic process

GO:0051704 Multi-organism process

Go:0051704 Metal ion homeostasis

Go:0009833 Plant-type primary cell wall biogenesis

Antimicrobial peptide 1

Metallothionein-like protein EMB30

Cysteine proteinase 15A

Antifungal protein ginkbilobin-2

Glycine-rich RNA-binding protein GRP1A

Malate dehydrogenase

Non-specific lipid-transfer protein 1

3-ketoacyl-CoA thiolase 2, peroxisomal

Isocitrate lyase

Catalase

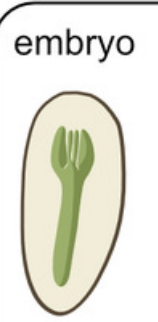

GO:000381 Regulation of alternative mRNA splicing

GO:0009451 RNA modification

GO:0015804 Neutral aminoacid transport

GO:0048508 Embryonic meristem development

GO:0006457 Protein folding

GO:0042254 Ribosome biogenesis

GO:0009813 Flavonoid biosynthetic process

GO:0009812 Flavonoid metabolic process

GO:0043170 Macromolecule metabolic process

GO:0031145 Anaphase-promoting complex-dependent catabolic process

Glycine-rich RNA-binding protein GRP1A

Cold shock domain-containing protein 4

60S ribosomal protein L36-1

Metallothionein-like protein EMB30

Translationally-controlled tumor protein homolog

Non-specific lipid-transfer protein

Chlorophyll a-b binding protein $1 \mathrm{~A}$, chloroplastic

Non-specific lipid-transfer protein 2

Ribulose bisphosphate carboxylase small chain, chloroplastic

60 S acidic ribosomal protein P1

\begin{tabular}{|l} 
phloem \\
Go:0002376 Immune system process \\
Go:0006468 Protein phosphorylation \\
Go:0006800 Oxigen and ROS metabolic process \\
Go:0006952 Defense response \\
Go:0010031 Circumnutation \\
Go:0010149 Senescence \\
Go:0016265 Death \\
Go:0023052 Signaling \\
Go:0023060 Signal transmission \\
Go:0030001 Metal ion transport
\end{tabular}

Metallothionein-like protein EMB30

Glycine-rich RNA-binding protein GRP1A

Non-specific lipid-transfer protein 4

Cysteine proteinase $15 \mathrm{~A}$

Translationally-controlled tumor protein homolog

17.8 kDa class I heat shock protein

Cold shock domain-containing protein 4

Catalase

Granule-bound starch synthase 1, chloroplastic/amyloplastic

Dormancy-associated protein 1 


\section{Table 2 (on next page)}

Number of significantly enriched GO terms

Table 2. Total number, number of significantly enriched GO terms, and number of nonredundant terms in $P$. sylvestris tissues. 
1 Table 2. Total number, number of significantly enriched GO terms, and number of non-

2 redundant terms in $P$. sylvestris tissues.

\begin{tabular}{|c|c|c|c|c|c|c|}
\hline & \multicolumn{3}{|c|}{$\begin{array}{l}\text { Tissue preferentially upregulated } \\
\text { genes }\end{array}$} & \multicolumn{3}{|c|}{ Tissue-specific genes } \\
\hline & Total & Significant & Non-redundant & Total & Significant & Non-redundant \\
\hline Bud & 15681 & 452 & 169 & 2019 & 137 & 65 \\
\hline Embryo & 17461 & 253 & 182 & 1178 & 75 & 50 \\
\hline Megagametophyte & 9690 & 306 & 123 & 1363 & 111 & 51 \\
\hline Needle & 25295 & 401 & 170 & 3818 & 169 & 81 \\
\hline Phloem & 16371 & 422 & 181 & 1249 & 58 & 40 \\
\hline
\end{tabular}

